\title{
Matching the Local and Cosmic Star Formation Histories
}

\author{
Igor Drozdovsky ${ }^{1,2}$, Andrew M. Hopkins ${ }^{3}$, Antonio Aparicio ${ }^{1}$, Carme Gallart ${ }^{1}$, and \\ the LCID team ${ }^{4}$ \\ 1 Instituto de Astrofísica de Canarias, C/Vía Láctea s/n, 38200, La Laguna, Tenerife, Spain \\ dio@iac.es \\ 2 Astronomical Institute of St.Petersburg State University, Russia \\ 3 University of Sydney, School of Physics, Bldg A28, NSW 2006, Australia \\ ahopkinsephysics.usyd.edu.au \\ 4 http://www.iac.es/project/LCID
}

Given the many recent advances in our understanding of the star formation history (SFH) of the Local Group and other nearby galaxies, and in the evolution of star formation with redshift, we present a new comparison of the comoving space density of the star formation rate as a function of look-back time for the Local and Distant Universe. We update the Local SFH derived from the analysis of resolved stellar populations ("fossil records") in individual nearby galaxies, based on our own estimations as well as available in the literature. While the preliminary comparison of SFHs is found to be broadly consistent, some discrepancies still remain, including an excess of the Local SFR density in the most recent epoch.

The goal of this project is to establish whether the star formation history (SFH) of galaxies in the local Universe is consistent with the cosmic (global) SFH inferred from surveys of distant, 'high-redshift', galaxies. A common approach based on redshift surveys aims to measure indicators of recent star formation in galaxies at different distances, which due to the finite speed of light, give us a view of different cosmic epochs. One of the drawbacks of this method is that it is not directly possible to connect different galaxies, measured at different redshifts, into a coherent evolutionary sequence, to provide a consistent picture of observed galaxy evolution. Moreover, the detail in which these galaxies can be studied is limited since they are mostly unresolved, and their faintness limits the wavelength resolution of any spectroscopic measurements. An alternative and complementary approach focuses on galaxies near enough to be resolved into their component stars. For these systems we can use well established stellar evolution theory, together with photometry and spectroscopy of individual stars of various ages, to interpret the "fossil record" of their star formation, and to trace the evolution of each from its formation to the present time. Despite a limited volume and sample size, the high quality SFHs one can obtain with this method is valuable in a general cosmological context. In par- 
ticular, it provides important clues to the earliest stages of star formation that are progressively harder to probe with galaxy redshift surveys targeting the most distant systems, as well as to probe issues that are not accessible through large-scale galaxy surveys (e.g., uncertainties in conversion factors from luminosity to SFR, dust attenuation, shape of the stellar and galaxy mass functions, etc). Initial comparisons suggest these different approaches do not yield the same results [12,10], but the errors are large, paradoxically, due to the lack of complete normalized SFHs of nearby galaxies.

New space- and ground-based multi-wavelength wide-field observational data, better understanding of stellar structure and evolution, and the recent development, coupled with greater computing power, of automated techniques that measure the star formation histories by statistical comparisons of models and observations [e.g., $13,1,4,8,7]$ have radically modified our view of star formation in the Milky Way (MW) and Andromeda (M31), the two gravitationally dominant galaxies of the Local Group [e.g., 14, 3]. Our knowledge of the star formation history in other nearby galaxies has also been significantly improved, thanks to the possibility of analysing their faint and ancient resolved stellar populations[e.g., 5, 6]. These low mass stars have extremely long lifetimes, comparable to the age of the Universe, and retain in their atmospheres the gas, with the elemental abundances intact, from the time of their birth. Thus they provide critical information on the early (initial) star formation rate and heavy element abundance. The total number of galaxies where these old ( $>10 \mathrm{Gyr}$ ) stars are measurable has doubled since the 2001 publication of Hopkins et al. [10].

In the past few years the measurement of the evolution of star formation rate (SFR) in various types of galaxies at a broad range of redshifts has also tremendously progressed. An example of an extensive compilation drawn from the literature of SFR density measurements at redshifts $0<z<6$ has been done by Hopkins and Beacom [9], following an investigation that carefully addressed issues associated with corrections for obscuration by dust within galaxies, internally consistent calibrations for the various star-formation rate indicators, and constraints on the initial distribution of stellar masses in a burst of star formation, among others. Taken together, the most recent and robust data indicate a compellingly consistent picture of the cosmic SFH constrained to within factors of about $\sim 3$. There is now growing evidence that the evolution is essentially flat beyond $z \sim 1$, however it is still unclear whether at $z>3$ the evolution flattens, declines or continues to increase.

On Fig. 1, we present an updated comparison of the comoving space density of the star formation rate as a function of look-back time for the Local Group (LG) and distant Universe. To measure the comoving space density for the LG, we integrated the best currently known SFHs for the galaxies within a volume defined by a 1.5 Mpc-radius sphere, each normalized by its current total star formation rate, similar to the Hopkins et al. [10] approach. For the MW and M31 we combined various estimations of SFH for each of galactic stellar components, such as disk, bulge, and halo.

The cosmic SFH (cyan shaded area) is taken from the compilation of Hopkins and Beacom [9], and scaled to ensure consistency with the Salpeter (1955) initial 


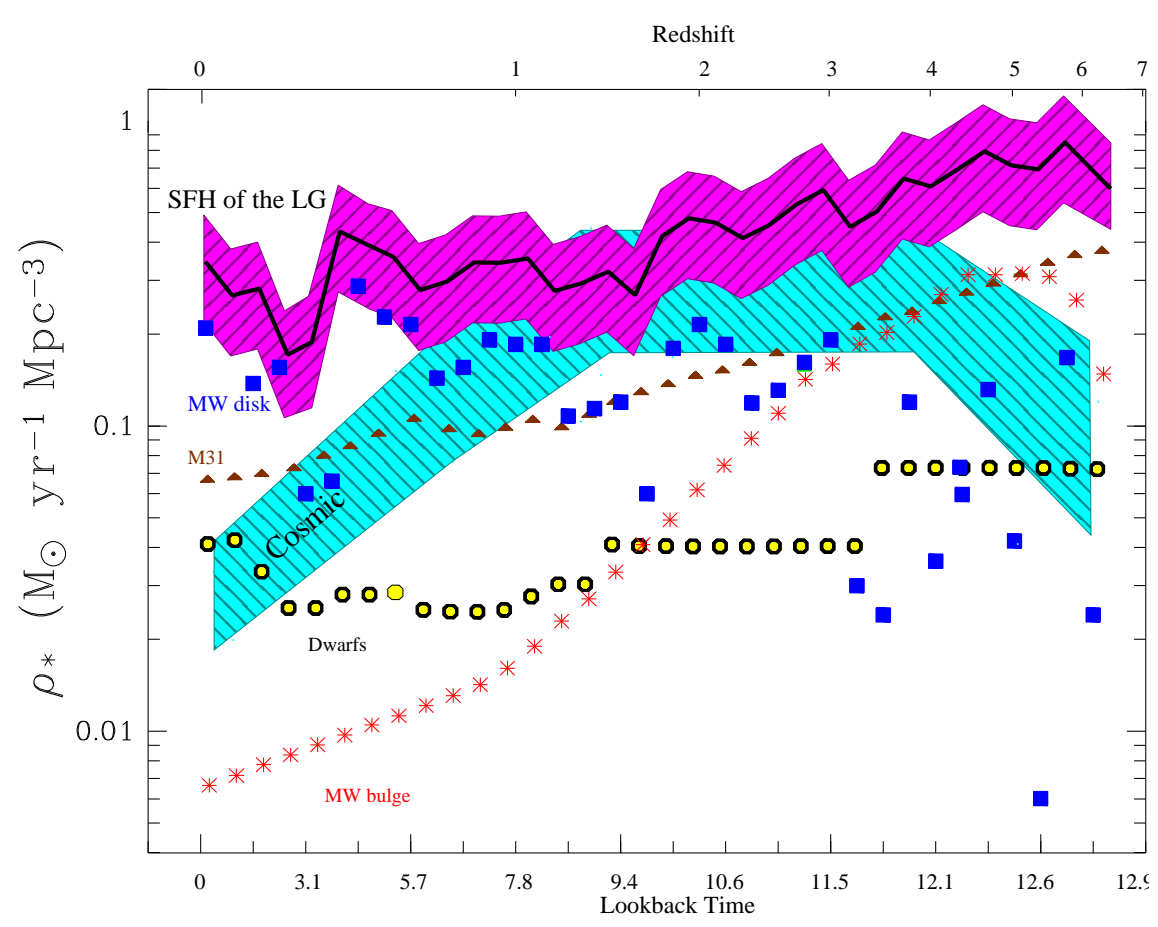

Fig. 1. Comparison of the Local Group SFH (solid line and magenta-shaded area) with that from a compilation of redshift surveys (cyan-shaded area) for a $\left(H_{0}, \Omega_{M}, \Omega_{\Lambda}\right)=$ $(70,0.3,0.7)$ cosmology. The squares, stars, triangles and circles show the contributions from the MW disk, MW bulge, M31 and dwarf galaxies respectively.

mass function. The shaded areas indicate the uncertainty in the star formation rate densities for the LG (dominated by uncertainties in the total star formation histories of the MW and M31), and for the cosmic star formation rate.

The results of this comparison are:

- An excess of the local star formation density in the recent $\sim 5$ Gyr is mainly due to the fluctuations of star formation of the MW disk.

- Between $\sim 8$ and $\sim 12 \mathrm{Gyr}$, the SFH of the LG is broadly consistent with the Cosmic one.

- The early/initial evolution of the LG was dominated by the spheroidal component of the MW and M31.

- The overall trend of $\rho_{*}$ from the LG supports a fairly flat evolution of the SFR, suggesting factor of $\sim 10$ extinction correction to high-redshift, UV-based SFR measures.

The important question is whether star formation in the Local Group was representative of the cosmic mean. Following an extension of the copernican principle, one can assume that the Local Group has no special location in the Universe and 

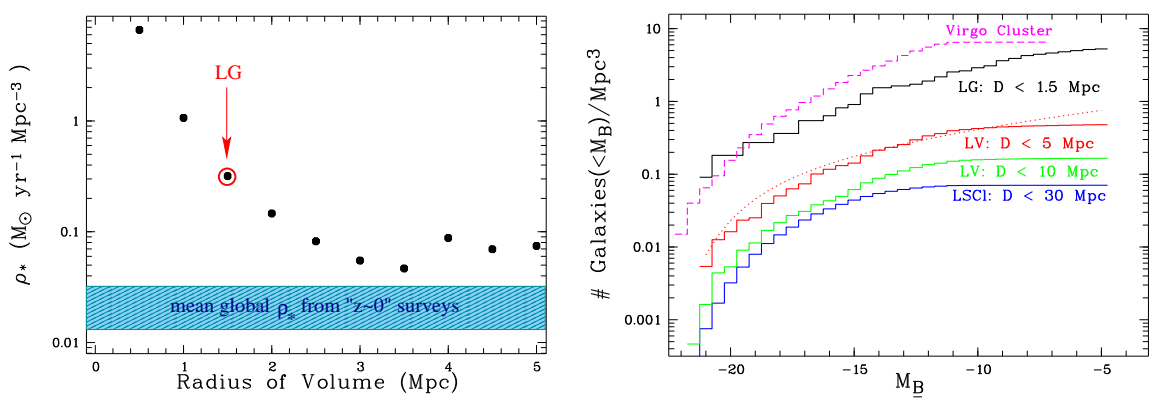

Fig. 2. Left: Current $(\mathrm{H} \alpha)$ star formation density as a function of volume size. Right panel: Comulative luminosity function (B band) for the different samples of galaxies within $30 \mathrm{Mpc}$.

hence its SFHs should be explained rather naturally, without appeal to special conditions. Certainly there are some pecularities of the LG environment from the larger volume, such as a dominance by two large spirals, an excess of the local current SFR density, and a deficiency of dwarf galaxies in comparison with denser environments (Fig. 2; Drozdovsky et al. 2008, in prep.). At the same time, about $85 \%$ of agalaxies are situated outside the dense environments of rich clusters, with roughly half of them belonging to groups like the Local Group, and the remaining half scattered in the 'field' $[11,2]$. An extension of the "fossil record" studies to a larger volume and improving our knowledge of the LG SFH will provide a more robust comparison of the Local and Cosmic evolution.

\section{References}

[1] A. Aparicio, C. Gallart, C. Chiosi, and G. Bertelli: ApJ, 469,L97 (1996)

[2] J. Bland-Hawthorn and K. C. Freeman: Mem. della SAI, 77,1095 (2006)

[3] T. M. Brown, E. Smith, H. C. Ferguson, et al.: ApJ, 652,323-353 (2006)

[4] A. Dolphin: New Astronomy, 2,397-409 (1997)

[5] A. E. Dolphin, D. R. Weisz, E. D. Skillman, and J. A. Holtzman: astro$p h / 0506430(2005)$

[6] C. Gallart and the LCID team: In Revista Mexic. de A\&A, volume 29, pages 158-158 (2007)

[7] J. Harris and D. Zaritsky: ApJS, 136,25-40 (2001)

[8] X. Hernandez, D. Valls-Gabaud, and G. Gilmore: MNRAS, 304,705-719 (1999)

[9] A. M. Hopkins and J. F. Beacom: ApJ, 651,142-154 (2006)

[10] A. M. Hopkins, M. J. Irwin, and A. J. Connolly: ApJ, 558,L31-L33 (2001)

[11] I. D. Karachentsev: AJ, 129,178-188 (2005)

[12] E. Tolstoy: astro-ph/9807154 (1998)

[13] E. Tolstoy and A. Saha: ApJ, 462,672 (1996)

[14] B. F. Williams: AJ, 126,1312-1325 (2003) 